\title{
Alfonso X el Sabio y La Mancha santiaguista
}

\author{
Ángela MAdRid y MEDINa *
}

El origen del Campo de Montiel, que llegó a alcanzar una superficie máxima de 3.458 kilómetros cuadrados ${ }^{1}$, lo encontramos en el castillo del mismo nombre y el terreno circundante. Con anterioridad a 1195 ya se había reconquistado Almedina, Albaladejo y Alcubillas, castillo éste que se donó en 1181 a la Orden de Santiago ${ }^{2}$. De todas maneras, y tras el duro golpe de Alarcos, no puede hablarse de repoblación hasta 1212. Con gentes del norte, mozárabes y, como en Villa Real ${ }^{3}$, judíos. En una tierra casi desértica. Estará a cargo de la mencionada Orden con un régimen señorial de matiz religioso.

Mediante sucesivos privilegios se le van entregando una serie de fortalezas. La de Eznavejor o Torres de Xoray ${ }^{4}$, de la que hoy sólo queda el testimonio de unas ruinas en el todavía llamado Estrecho de las

* UNED, Valdepeñas.

Manuel Corchado y Soriano, Avance de un estudio geográfico-histórico del Campo de Montiel, Madrid, IEM, 1971, pág. 8.

2 "... castrum Alcabelam cum pertenentiis suis et hereditates alias quas de largitione hermani in Christo filli nostre..." $A H N$ Ordenes Militares, carp. 214, núm. 1.

${ }_{3}$ Puede verse Luis Delgado Merchan, La judería y la Inquisición de Ciudad Real, Ciudad Real, Imprenta del Comercio de José María Vega, 1893.

${ }_{4}$ “Ego ALDEFONSUS Dei gratia rex Castelle... libenti animo et uoluntate spontanea pro remedio anime mei et parentum meorum... facio cartam donationis, concessionis, confirmationis et stabilitatis Deo et ordini milicie beati Jacobi et uobis dono Garsie Gonzal ui eiusdem ordinis militaris magistro uirisque sucessoribus et uniuerso eiusdem ordinis fratrem conuentui presenti futurique perpetuo ualituram. Dono itaque uobis et concedo castellum illud quod dicit Feznauexore quod ego acquisiui per Dei clemencia a paganis... cum omnibus terminis suis montibus nemoribus aquis riuis pratis pascuis et omnibus pertenentis suis..." AHN Ordenes Militares, carp. 214, núm. 2. 
Torres, entre Villamanrique y Torre de Juan Abad, el 3 de mayo de 1214. Unos años después, el 15 de marzo de 1227 en Carrión, Fernando III el Santo hace donación de San Polo y Montiel al maestre Pedro González ${ }^{5}$. Del castillo de San Polo no queda nada en la actualidad. Frente a éste el de la Estrella que, aunque ha corrido mejor suerte y en 1478 permanecía en aceptable estado de conservación ${ }^{6}$, ha sufrido el paso del tiempo con una irreversible crueldad. Cuando se redactan las Relaciones Topográficas de Felipe $1{ }^{7}$ ya presentaba muestras indudables de abandono. Se había hundido la cocina, las caballerizas y diversas dependencias. La iglesia, sin embargo, debia de hallarse bien conservada, albergando una imagen de alabastro del Apóstol Santiago. Destaca la bóveda «muy principal».

El maestre Pelayo Pérez Correa, por lo demás rigurosamente contemporáneo de Alfonso X - su maestrazgo abarca de 1242 a 1275otorgó a Montiel en 1243 el fuero de Cuenca ${ }^{8}$ seguido de una serie de privilegios $^{9}$.

La cuestión no se agota en la Edad Media. En 1598, por ejemplo, observamos que con motivo de realizarse en Villanueva de los Infantes

${ }^{5}$ “... Ego FERDINANDUS Dei gratia rex Castelle... facio cartam donationis concessionis confirmationis et stabilitatis Deo et ordini milicie beati Jacobi uobis dono Petro Gonçalui... et sucessoribus uestris et uniuerso fratrem conuentui presentibus et futuris dono itaque uobis et concedo sanctum Paulum et Montellum cum omnibus terminis suis montibus fontibus riuis pratis pascuis molindinis et eorum locis cum ingressibus et egressibus et omnibus pertenentiis suis..." AHN carp. 214, núm. 6 .

'AHN OO.MM. Visitas, $1063 \mathrm{c}$.

' C. VINAS y R. PAZ. Relaciones de los pueblos de España ordenadas por Felipe II. Ciudad Real, Madrid, C.S.I.C., 1971, págs. 346 y 347.

\& «... damos... al concejo de Montiel, á la Villa, é á las Aldeas, todo él, é sus términos, el fuero de Cuenca,... damosle la tercia parte de los Montadgos; é todas otras cosas que ahi son,...; é este fuero los damos por todos los tiempos per secula seculorum...". Inocente Hervas y Buendia, Diccionario histórico-geográfico de la provincia de Ciudad Real, Ciudad Real 1890, pág. 352.

s En 1261: “que mancebo, é manceba, que nunca fueren casados, é casaren de primas en Montiel, é en su termino no pechen por un año... Ibidem, pág. 353. Y en 1268: "... otorgarnos á el Concejo de Montiel, tambien á la villa, como á las aldeas... que no laste... el marido por la muger, ni la muger por el marido, ni padre por el hijo, ni el hijo por el padre; é... que tomen en cada lugar sendos desmeros de los mejores que ahi fueren por ayuda á sus Iglesias, esto todo lo hayan para siempre:... que don Lope Hernandez (el Comendador) no mampare casa ninguna, sino aquella en que el morare:... que ningun home sea osado,... de prender ladron, ni mal-hechor, ni otro home; sino fuere el Concejo, con las Justicias de la Villa:.. la mitad del montadgo... que corten, é que pazcan con los de la Ossa,... que las Dehessas, que sean assi, como las mojonó Gonzalo Ruiz de Rojas, con los otros buenos homes... mandamos al Concejo de la Villa de Montiel, la Dehessa de los Conejos de Montiel por suya..." Ibidem, pág. 354 
un reclutamiento de soldados, se indica que «podria ser que oviese algunas ciudades, villas y lugares donde por previlexios de los señores reyes mis progenitores y mios no ubiesse padrones de hijos dalgos ni pecheros por raçon de las libertades y exenciones que en los tales previlexios se les concede, es mi voluntad que en estos no se proçeda por la forma y orden arriva declarada en quanto a hazer repartimiento del diez uno entre los buenos hombres pecheros del número que faltare sobre los que su voluntad se ubieren asentado, sino que la justiçia e regimiento con vuestra yntervençion los señale y supla en la forma que mas pareçiera convenir..." ${ }^{10}$ y todavia, el 17 de diciembre de 1744 , tenemos un privilegio de Felipe $V$ renunciando, previo pago de 30.000 reales de vellón que hizo efectivo el concejo de Montiel, al derecho que el patrimonio real tenía sobre la aldea despoblada de Torres con sus términos, hierbas, dehesas y todos los demás aprovechamientos, que, por otro lado, Montiel venía disfrutando ya como propios ${ }^{11}$.

Este es el contexto en el que podemos inscribir los documentos otorgados por Alfonso $X$ siendo aún príncipe, aparece la donación de Villanueva (de la Fuente) y Gorgogi a la orden de Santiago ${ }^{12}$. Estos territorios ya fueron objeto de litigio con el concejo de Alcaraz que en 1242: «... se embio querellas por sos bonos omes a mi don Ferrando... de los freyres de la caualleria de sant Jague que se ies entregaran sos terminos que les fueran dados del ondrado rey don Alfonso, myo auueio, et que auien hy poblado castiellos et aldeas. Los nombres de los quales castiellos et aldeas son estos que aqui son escriptos: Villanueua, Gorgogi, Villafranca, Albaladeio, Borialista, Terrinches, El Finoio, Santa Marina, La Fuent el Mayello, Odes, El Almedina, La Fuent de la Figuera, la Torre de Johan Abbat, Cernina, Jamila, Peña Flor, Alcobiella, Mont Agudiello, Las Nauas de la Condessa, Torres, La Fuent Plana, La Sarça, Cañamares, Cañamareio, Turra, El Salediello, La Carrizosa, El Aliezira, La Roydera, El Tocon, la Membriella, El Carrizal, Quitrana, Posadas Vieias, Villaaios, Migael Esteuan, Almuradiel, La Figuera, El Cueruo, Villareio Ruuio, Mania Vacas. Et estas querellas me dieron los del Alcaraz delant el Maestro que era a la sazon et ante el Comendador et ante otros muchos freyres et ellos diyieron que estos terminos et estos logares que los de Alcaraz demandauan que de la orden eran et que mostrarien dello bonos priuile-

${ }^{10}$ Archivo Municipal de Villanueva de los Infantes, carp. 1, legajo 2 , fol. $4 \mathrm{v}$ y $5 \mathrm{r}$. La catalogación de este archivo no es definitiva.

11 Archivo Municipal de Montiel. Sin catalogar.

${ }^{12}$ AHN Órdenes Militares, carp. 365, núm. 5. 
gios et bonas cartas que tenien... despues que oluiemos uistas las cartas de la una part et de la otra et despues de muchos razonamientos todo el pleyto fue librado desta guisa: que los freyres se partieron de Villanueua et de quanto derecho hy auien et cuydauan auer et de la heredat de Gorgori et de quanto derecho hy auien et deuien auer et dexaronlo en la mi mano suelta mientre et de plano para darlo a los de Alcazar o ser dello lo que yo quisiesse. Et yo do al concecio de Alcaraz por heredamiento... Villanueua por su aldea entregada mientre con su torre et con todos sus terminos laurados et por laurar et con entradas et con eyidas et con montes, con fuentes, con pastos et con todas sus pertenencias. Et este es el termino de Villanueua a la parte de Montiel que de Villanueua fasta Montiel que la quarta parte sea termino de Villanueua et las tres partes sean termino de Montiel... A la parte de Albaladeio es termino de Villanueua assi como el maestro et los freyres deuisaron et departieron termino para Villanueua et assi como los de Villanueua laurarian et tenien el dia que esta carta fue fecha... et de la otra parte como toma del moion que es entre Villanueua et Montiel et como ua derechamientre a Turra et seer en Turra el un moion et Turra es de Alcaraz et desend adelant a la suerte del Puerto el otro moion et nenguno non la pueble et dend adelant es moion la Laguna de Medio. Otrosi do yo por heredamiento a los de Alcaraz el heredamiento que los freyres auien en Gorgogi que lo ayan libre et quito por siempre... Otrosi do et otorgo et confirmo a Dios et a la orden de la caualleria de sant Jague todos aquellos logares que de suso son nombrados de que los de Alcaraz se me auien querellado que los aya siempre liures et quito sin contradicho nenguno con todos sus terminos et sus pertenencias que nin yo nin nenguno de myos successores ni los de Alcaraz nunqua mas podamos demandar cosa nenguna nin mouerles pleyto nenguno sobrellos. Et yo porque la orden et los de Alcaraz ayan siempre abenencia et paz entre si, mando et establesco que buian desta guisa que en sus montes et en sus sierras ayan siempre uso de pacer et de cortar comunalmientre los unos en lo de los otros sacadas end dos defesas... ${ }^{13}$ ".

Tras una serie de presiones, el documento está fechado el 18 de febrero en Valladorin. Algo después, el 11 de septiembre, en Burgos, san Fernando otorga a la orden de Santiago la iglesia de Villanueva ${ }^{14}$.

La villa junto con Gorgogi, que a tal efecto se adquirió por compra, fueron entregadas como ya hemos indicado, por el príncipe Alfonso el 13

\footnotetext{
${ }^{13}$ AHN Ordenes Militares, carp. 365, núm. 2.

${ }^{14}$ AHN Ordenes Militares, carp. 365, núm. 4.
} 
de agosto de 1248 a P. Correa. Ello clarificaba situaciones legales. Sin embargo, los conflictos con Alcaraz no quedaron zanjados de manera definitiva. $Y$ ello obliga al rey en 1263 a salir nuevamente al paso encargando a cinco «hombres buenos» que realicen una detallada señalización en términos ${ }^{15}$. Con todo, ni la cuestión de Villanueva, ni los pleitos sobre términos en el Campo de Montiel, quedan liquidados. Sobre el primer caso existe otro privilegio, esta vez de 1369, por el que Enrique II entrega a "don Gonçalo Mexia maestre de la cavalleria de la orden de Santiago, los muy altos et muy grandes et muy señalados serviçios que vos el dicho et la dicha vuestra orden nos fezistes et avedes fecho et fazedes de cada dia señaladamiente por quanto... vos açercastes... contra pelea que ovimos agora cerca de Montiel quando vençimos et desvaratamos aquel malo tirano que se llamava rey. Por ende por vos dar galardon de los dichos serviçios et por quanto afan et travajo avedes parado por nuestro serviçio damosvos en donaçion pura et non revocable por juro de heredat... el nuestro lugar de Villanueua, aldea de Alcaraz, con todos sus terminos et pertenençias...” ${ }^{16}$.

Por lo demás, en lo que a términos se refiere los pleitos se prolongan en el tiempo en diversos lugares del Campo, como puede verse en la documentación del archivo de la Cancillería de Granada ${ }^{17}$ y en algunos locales ${ }^{18}$.

Lo pasajero del territorio y el deseo de atraer nuevos pobladores, ya, sin duda, mucho más inclinados a las fértiles tierras andaluzas, mueve al rey a conceder una feria a Montiel ${ }^{19}$ en 1252 . Se debía de iniciar el día de San Lucas y su duración (inferior a la habitual) era de diez dias. Si bien es cierto que no conocemos su trayectoria, ni la repercusión que tuvo en el Campo, lo que nos lleva a pensar que fue escasa, tiene de peculiar el ser la primera de su reinado. La otorga por la ayuda que la Orden de Santiago le prestó en la conquista de Murcia.

15 Apéndice, núm. Ill.

${ }^{16}$ AHN Órdenes Militares, carp. 365, núm. 8. Viene inserto en una confirmación de Juan I.

17 Algo de ello recojo en el libro Ciudad Real en el archivo de la Chancillería de Granada, Ciudad Real, IEM, 1983.

${ }^{18}$ En 1755 hay un conflicto del concejo de Villahermosa con los ganaderos de Infantes sobre propiedad y configuración de las dehesas del término. Archivo Municipal de Villahermosa. Sin catalogar.

19 Apéndice I. 
Cuatro años más tarde también quiso agradecer la colaboración, en este caso del comendador de Segura, sobre Orihuela, entregándole Abeiazat, origen del actual Socuéllamos ${ }^{20}$. Y aunque trascienda los limites estrictos del Campo de Montiel, nos ha parecido necesario recoger este último privilegio por su interés y por globalizar la actuación de Alfonso $X$, a quien la fundación de Villa Real no le impidió ocuparse de las Ordenes Militares. Santiago en este caso.

${ }^{20}$ Apéndice II. Puede verse al respecto mi articulo "La encomienda de Socuéllamos" y «El virrey Mendoza» publicado por Surcos, Ciudad Real 1986. 


\section{APÉNDICE DOCUMENTAL}

1252, agosto, 8. Sevilla

Privilegio real de Alfonso $X$ autorizando la celebración de una feria en Montiel por la ayuda que el maestre Pérez Correa le prestó en la conquista de Murcia

A.H.N. Órdenes Militares. Carp. 214. n. ${ }^{\circ} 11$.

(Chrismón, alfa y omega). Connosçida cosa sea a quantos esta carta uieren como yo don ALFONSO por la gracia de Dios rey de Castiella de Toledo de Leon de Gallizia de Seuilla de Cordoua de Murcia et de Jahen en uno con la reyna doña Yolant mi mugier do et otorgo a la orden de la Caualleria de Sanciague et a vos don Pelay Perez maestro dessa misma orden et a todos los otros maestros que seran despues de uos en esta orden sobredicha por seruitios que me fiçciestes en la conquista de Murcia et me faredes, que fagades feria en Montiel et que comience el dia de Sant Lucas et que dure diez dias. Et do uos la que la ayades por siempre iamas con su portadgo et con todos sus derechos assi como la ha el arçobispo et la Eglesia de Toledo en Santyuste de Alcala saluo ende el mio sennorio que la mande yo guardar et que aya la meatad de las calonnas que fizieren en la feria yo et todos aquellos que regnaren despues de mi en Castiella et en Leon. Et mando a todos aquellos que uenir quisieren a esta feria que uengan saluos et seguros por todo mio regno et por todo mio sennorio con todas sus cosas et todo sus derechos o darlos deuieren et non sacando cosas uedadas del Regno nenguno non sea osado de los fazer fuerça nin tuerto nin mal nenguno ca qualquiere que lo fiziesse auie mi yra et pecher mie en coto mil marauedis et a ellos todos el danno doblado. Et porque este mio donadio uala para siempre mande poner el mio sello de plomo en esta carta. Et yo sobredicho rey don ALFONSO regnando en Castiella en Toledo en Leon en Galizia en

" Lo menciono en "El Campo de Montiel en la Edad Moderna" Cuaderno de Estudios Manchegos (1979) págs. 26 y 27. 
Seuilla en Cordoua en Murcia en Jahen en Badaioz en Baeça et en el Algarue otorgo et confirmo esta mi carta. Fecha la carta en SeuiIla por mandado del rey, VIII dias de agosto era de mill et dozientos et nonaenta annos.

Don Alfonso de Molina confirma, don Frederic confirma, don Enrique confirma, don Manuel confirma, don Fernando confirma, don Felippe electo de Seuilla confirma, don Sancho electo de la Eglesia de Toledo confirma, don Aboabdille Auenaçar rey de Granada et uasallo del rey don Alfonso confirma.

(1. $\left.{ }^{a} \mathrm{col}.\right)$

Don Apparicio, obispo de Burgos, confirma.-Don Rodrigo, obispo de Palencia, confirma.-Don Remondo, obispo de Segouia, confirma.-Don Pedro, obispo de Siguença, confirma.-Don Gil, obispo de Osma, confirma.-Don Mathe, obispo de Cuenca, confirma.-Don Benito, obispo de Auila, confirma.-Don Aznar, obispo de Calaforra, confirma.-Don Adam, obispo de Plazencia, confirma.-Don Loppe, obispo de Cordoua, confirma.-Don Pascual, obispo de Jahen, confirma.-Don Pedro, obispo de Cartagena, confirma.-Don Pelay Correa, maestro de la orden de Ucles, confirma.

(2. $\left.{ }^{\mathrm{a}} \mathrm{col}.\right)$

Don Nunio Gonzalez, confirma.-Don Alfonso Lopez, confirma.-Don Rodrigo Gonzalez, confirma.-Don Symon Royz, confirma.-Don Alfonso Tellez, confirma.-Don Fernando Royz de Castro, confirma.-Don Pedro Nuñez, confirma.-Don Nunio Guillerm, confirma.-Don Pedro Guzman, confirma.-Don Rodrigo Gonzalez el Niño, confirma.-Don Gomez Royz, confirma.-Don Fernand Garcia, confirma.-Don Alfonso Garcia, confirma.-Don Rodrigo Aluarez, confirma.-Don Diago Gomez, confirma.-Don Pedro Lopez de Harana, confirma.

(SIGNO RODADO. LEYENDA): signun el rey Aldefonsus.

(EN TORNO AL SIGNO): Don Johan Garcia, mayordomo de la Corte del Rey, confirmo.-Don Diago Lopez del Faro, alferiz del Rey, confirmo. 
(3. $\left.{ }^{a} \mathrm{col}.\right)$

Don Johan, arzobispo de Sanciague, confirma.-La Eglesia de Leon Uacat.-Don Pedro, obispo de Ouiedo, confirma.-Don Pedro, obispo de Astorga, confirma.-Don Pedro, obispo de Çamora, confirma.-Don Pedro, obispo de Salamanca, confirma.-Don Migael, obispo de Lugo, confirma.-Don Johan, obispo de Orens, confirma.-Don Johan, obispo de Mondoñedo, confirma.-Don Gil, obispo de Tui, confirma.-Don Leonardo, obispo de Cipdat, confirma.-Don Sancho, obispo de Coria, confirma.-Don Fernando Ordoñez, maestro de la Orden de Calatrava, confirma.

(4. $\left.{ }^{\mathrm{a}} \mathrm{col}.\right)$

Don Rodrigo Alfonso, confirma.-Don Martin Alfonso, confirma.-Don Rodrigo Gomez, confirma.-Don Rodrigo Frolaz, confirma.-Don Fernand Yuañez, confirma.-Don Martin Gil, confirma.-Don Johan Perez, confirma.-Don Andreo, perteguero de Sanciague, confirma.-Don Gonzaluo Ramirez, confirma.-Don Rodrigo Rodriguez, confirma.-Don Ramil Diaz, confirma.-Don Aluar Diaz, confirma.-Don Pelay Perez, confirma.-Don Gonzaluo Morant, confirma.-Don Fernand Gonzalez, merino mayor, confirma.-El Merinazgo de Leon uacat.-Don Moño Fernandez, merino mayor en Gallizia, confirma.-Maestro Fernando, notario del rey en Castilla, confirma.-Don Martin Fernandez, notario del rey en Leon, confirma.-Escriuiol Sancho Fernandez, escribano del rey. 
1256, julio, 5. Segovia

Privilegio real de Alfonso $X$ otorgando, por la ayuda prestada en la conquista de Orihuela, al comendador de Segura y a la Orden de Santiago el cortijo de Abeiazat.

A.H.N. Órdenes Militares. Cajón 315. n. ${ }^{\circ} 1$.

(Chrismon, alfa y omega). Connosçida cosa sea a todos los omies que esta carta uiren cuemo yo don ALFONSO por la gracia de Dios rey de Castiella de Toledo de Leon de Gallizia de Seuilla de Cordoua de Murcia et de Jahen en uno con la reyna doña Yolant mi mugier et con mio fijo el Infante Ferrando por grant fabor que he de fazer bien et mercet a don Pedro Fernandez comendador de Segura et sennaladamientre por el seruicio que me fizo sobre Orihuela quando la gane do et otorgo a el et a la Orden de Ucles a los que agora son et seran de aqui adelante pora siempre iamas el cortijo que dizen Abeiazat que es en La Mancha. Et este cortijo sobredicho les do et les otorgo con montes con fuentes con rios con pastos con entradas con salidas et con todas sus pertinencias assi cuemo las ha et las deue auer que lo ayan libre et quito por iuro de heredat pora siempre iamas pora dar pora uender pora empennar pora canuiar pora enagenar et pora fazer dello et en ello todo lo que ellos quisieren cuemo de los suio mismo. Et tengo pora $\mathrm{mi}$ et pora todos aquellos que regnaren despues de mi en Castiella et en Leon moneda et que entre Ley el mio adelantado pora fazer iusticia assi cuemo entra en todos los otros logares de la Orden et que me fagan del guerra et paz a mi et a todos aquellos que regnaren despues de mi en Castiella et en Leon. Et mando et defiendo que ninguno non sea osado de yr contra este priuilegio deste mio donadio ni de quebrantarle ni de minguarle en ninguna cosa ca qualquier que lo fiziesse aurie mi yra et pechar mie en coto mill morauedis et al comendador et a la Orden sobredicha todo el danno doblado. Et porque este priuilegio sea firme et estable mandolo seellar con mio seello de plomo. Fecha la carta en Segouia por mandado del rey $V$ dias andados del mes de julio en era de mill et dozientos et nonaenta et quatro annos. Et yo sobredicho rey don Alfonso regnant en uno con la reyna donna Yolant mi mugier 
et con mio fijo el infante don Ferrando en Castiella en Toledo en Leon en Gallizia en Seuilla en Cordoua en Murcia en Jahen en Baeça en Badalloz et en el Algarue otorgo este priuillegio et confirmolo.

Don Sancho electo de Toledo et chanceler del rey confirma. Don Felip electo de Seuilla confirma. Don Nunio Gonzaluez confirma. Don Alfonso Lopez confirma. Don Symon Royz confirma. Don Aboabdille Abennaçar, rey de Granada, uasallo del rey confirma. Don Alfonso fijo del rey Johan enperador de Constantinopla et de la enperadtriz donna Berenguella comde Do uassallo del rey confirma. Don Loys fijo del enperador et de la enperadtriz sobredichos conde de Belmont uassallo del rey confirma. Don Johan fijo del enperador et de la enperadtriz sobredichos conde de Montfort uassallo del rey confirma. Don Mahomat Abenmafomat Abenhuth rey de Murcia uassallo del rey confirma. Don Gaston bizconde de Beart uassallo del rey confirma. Don Gui bizconde de Limoges uassallo del rey confirma. Don lohan arçobispo de Sanctyago et chanceller del rey confirma. Don Manuel confirma. Don Ferrando confirma. Don Loys confirma. Don Abenmafoth rey de Niebla uassallo del rey confirma.

\section{(1. $\left.{ }^{\mathrm{a}} \mathrm{col}.\right)$}

Don Apparicio obispo de Burgos confirma. Don Ferrando obispo de Palencia confirma. Don Remond obispo de Segouia confirma. Don Pedro obispo de Siguença confirma. Don Gil obispo de Osma confirma. Don Mathe obispo de Cuenca confirma. Don Benito obispo de Auila confirma. Don Aznar obispo de Calahorra confirma. Don Lop electo de Cordoua confirma. Don Adam obispo de Plazencia confirma. Don Pasqual obispo de lahen confirma. Don frey Pedro obispo de Cartagena confirma. Don Pedriuannez maestre de la Orden de Calatrava confirma.

(2. $\left.{ }^{\mathrm{a}} \mathrm{col}.\right)$

Don Alfonso Tellez confirma. Don Ferrand Royz de Castro confirma. Don Pedro Nunnez confirma. Don Nunnio Guillem confirma. Don Pedro Guzman confirma. Don Rodrigo Gonzaluez el Ninnio confirma. Don Rodrigo Alvarez confirma. Don Ferrand Garcia confirma. Don Alfonso Garcia confirma. Don Diago Gomez confirma. 
Don Gomez Royz confirma. Don Gutur Suarez confirma. Don Suer Tellez confirma.

(SIGNO RODADO. LEYENDA): El rey don Alfonso signoo.

(ENTORNO DEL SIGNO): Don Juan Garcia mayordomo de la corte del rey la confirma. El alferizia del rey uaca.

(3. $\left.{ }^{a} \mathrm{Col}.\right)$

Don Martin obispo de Leon confirma. Don Pedro obispo de Ouiedo confirma. Don Suero obispo de Çamora confirma. Don Pedro obispo de Astorga confirma. Don Leonard obispo de Cibdat confirma. Don Miguel obispo de Lugo confirma. Don Johan obispo de Orens confirma. Don Gil obispo de Tuy confirma. Don Johan obispo de Mondonedo confirma. Don Pedro obispo de Coria confirma. Don frey Rober obispo de Silue (?) confirma. Don frey Pedro obispo de Badalloz confirma.

(4. ${ }^{\mathrm{a}} \mathrm{col}$.)

Don Alfonso Ferrandez fijo del rey confirma. Don Rodrigo Alfonso confirma. Don Martin Alfonso confirma. Don Rodrigo Gomez confirma. Don Rodrigo Frolaz confirma. Don Johan Perez confirma. Don Ferrando Yuannes confirma. Don Martin Gil confirma. Don Gonzaluo Ramirez confirma. Don Rodrigo Rodriguez confirma. Don Aluar Diaz confirma. Don Pelay Perez confirma. Don Pelay Perez maestre de la Orden de Sanctiago confirma. Don Garci Ferrandez maestre de la Orden de Alcantara confirma. Don Martin (borrado) maestre de la Orden del Temple confirma. Garci Suarez merino mayor del regno de Murcia confirma. Don Ferrando obispo de Palencia notario del rey en Castiella confirma. Roy Lopez de Mendoça almirante de la mar confirma. Sancho Martinez de Xodar adelantado de la frontera confirma. Garci Perez de Toledo notario del rey en el Andaluzia confirma. Gonzalo Morant merino mayor de Leon confirma. Roy Suarez merino mayor de Gallizia confirma. Don Suero obispo de Camora notario del rey en Leon confirma. (borrado) Garcia de Fromesta la escriuio el anno quarto que el rey don Alfonso regno. 


\section{3 *}

1263, marzo, 22. Sevilla

Carta abierta de Alfonso $X$ por el que sale al paso de las discrepancias existentes entre la Orden de Santiago y el concejo de Alcaraz sobre delimitación de términos

A.H.N. Órdenes Militares. Carp. 214. n. ${ }^{\circ} 17$.

Sepan todos los omes que esta carta vieren cuemo nos don ALFONSO por la gracia de Dios rey de Castiella de Toledo de Leon de Gallizia de Seuilla de Cordoua de Murcia de Jahen et del Algarbe otorgamos que sobre contienda que era entre don Pelay Perez maestre de la orden de la caualleria de Sanciago por si et por su orden et por sus uasallos et el concejo de Alcaraz de villa et de aldeas en razon de sus terminos et de otras querellas que auien los unos de los otros uinieron ante nos don Pelay Perez el maestre aobredicho et Migael Perez et Gil de Moya et Johan Matheo de Villanueua et Johan Donate personas del concejo de Alcaraz sobre que fueron aplazados por razonar estos pleytos los unos con los otros. Et antes que en pleyto entrasen auinieronse a mas las partidas plaziendo a nos que todos los pleytos et todas las demandas et todas las querellas que auien los unos de los otros en razon de los terminos et todas las otras cosas que lo metien en mano destos cinco omes bonos. El maestre de Ucles tomo por si et por su Orden et por sus uasallos Pedro Gutierrez comendador de Segura et Lope Ferrandez de Montiel sus freyres. Et estos personeros del concejo tomaron por si et por el concejo de Alcaraz de villa et de aldeas Migael Perez et Alfonso Gomez uecinos de Alcaraz et nos diemos hy de nuestra parte Ferrand Perez de Cuenca nuestro alcalde. Que estos cinco omes bonos sobredichos que uayan et uean los terminos de Alcaraz et de la Orden sobre que han las contiendas et alli do mostreren que tienen priuilegios cada una de las partes que pongan hy los moiones segund que diyieren los priuilegios et do

* Lo utiliza en «El Campo de Montiel», obra citada, pág. 21.

Con posterioridad Aurelio Pretel lo incorpora en Conquista y primeros intentos de repoblación del territorio albacetense. IEA. Albacete 1986, págs. 269-70. 
fallaren que no ay puestos moiones que los pongan en aquellos lugares entre el termino de Alcaraz et la Orden alli para mayor uerdat fallaren que deven seer. Et de los terminos que no pudieren fallar uerdat pero fueron los mojones que estos cinco sobredichos partan los terminos et pongan los mojones por aquellos lugares que ellos entendieren que sera con guisa et con razon. Et si por auentura todos estos omes sobredichos non se abiniessen en deparar esto assi como dicho es abinieronse que o la mayor prenda destos omes se otorgaren que sea firme lo que ellos ficieren et otrossi todas las otrs querellas que han los unos de los otros pusieron que estos omes sobredichos que las sepan de la una parte et de la otra et las fagan emendar et endereçar en aquella guisa que estos omes - la mayor parada dellos se andaren et tomaren por bien et otrossi abinieronse a mas las partes que el maestre nin su Orden nin sus uasallos nin el concejo de Alcaraz de uillas et de aldeas non fagan otras deffesas si non assi como manda el priuilegio del rey don Ferrando nuestro padre et pidieronos merced a mas las partidas que esta abenencia que fizieron assi como dicho es que nos plogiesse et que lo mandassemos assi fazer et complir et nos por su ruego et por sacar contienda entrellos et porque entendemos que sera nuestro seuicio et en pro dellos otorgamos esta abenencia assi como dicho es et mandamos a Ferrand Perez de Cuenca nuestro alcalde et a Pedro Gutierrez comendador de Segura et a Lope Fernandez de Montiel freyres de la orden de Ucles et a Migael Perez et a Alfonsso Gomez uezinos de Alcaraz que uayan de partir los terminos entre Alcaraz et la orden de Ucles et que fagan emendar et endereçar todas las otras querellas que han los unos de los otros assi como dicho es de guisa que lo hayan fecho et acabado todo por la Pascua de la quincuagesima esta primera que uiene que sera en la era desta carta et de como lo fizieren que nos lo enuien escripto et seellado con sus seellos et nos seguir de aquello mandaremos dar a cada una de las parten mas priuilegios et qualquier de las partidas que non quisiessen fincar por quanto estos omes sobredichos fiziessen assi como dicho es pechar nos ye la pena que dize el priuilegio del rey don Ferrando nuestro padre et a la otra partida quinientos marauedis et el danno doblado et de mas mandariemos que fincassen por quanto estos cinco sobredichos fiziessen. Et porque esto non uenga en dubda daremos esta nuestra carta abierta seellada con nuestro seello a estos cinco sobredichos. Fecha la carta en Seuilla por mandado del rey yueues XXII dias andados de marzo era de miil et trezientos et un anno. Yo Garcia Dominguez la fiz escreuir. 